\title{
Scrub typhus with multi-organ dysfunction syndrome and immune thrombocytopenia: a case report and review of the literature
}

\author{
Weijia Li, Lei Huang ${ }^{*}$ (D) and Weixing Zhang*
}

\begin{abstract}
Background: Scrub typhus is an acute infectious zoonotic disease caused by Orientia tsutsugamushi. Multi-organ dysfunction secondary to scrub typhus is hard to diagnose and has a high mortality rate. Only one case of scrub typhus with multi-organ dysfunction syndrome and immune thrombocytopenia has been reported thus far. In this study, we report a second case of scrub typhus with multi-organ dysfunction syndrome and immune thrombocytopenia, and we summarize its diagnosis and treatment.

Case presentation: A 43-year-old Han Chinese woman, a sanitation worker, was admitted to our hospital after 7 days of a skin infection and 5 days of a sore throat with fever and dizziness. A physical examination revealed the presence of an eschar on the right side of her neck. She had a history of insect bites during her sanitation work. A diagnostic evaluation identified scrub typhus as the primary illness, which was associated with multi-organ dysfunction syndrome and immune thrombocytopenia. She recovered completely after 15 days of treatment and extensive symptomatic supportive care.
\end{abstract}

Conclusion: We report a second case of tsutsugamushi disease with multi-organ dysfunction syndrome and immune thrombocytopenia, which resolved after treatment and extensive care.

Keywords: Scrub typhus, Multi-organ dysfunction syndrome, Immune thrombocytopenia

\section{Introduction}

Scrub typhus is a rickettsial zoonotic disease transmitted by mites and caused by Orientia tsutsugamushi [1]. Scrub typhus is a severe public health problem that affects mainly Asia-Pacific areas; scrub typhus threatens one billion people and causes illness worldwide each year [2]. The original name of scrub typhus, which was given by Hashimoto in 1810, is "tsutsugamushi disease." Although the median mortality rate of untreated patients is $6 \%$ and that of treated patients is $1.4 \%$, the mortality rate of scrub typhus remains high and can reach up to $70 \%$ without proper treatment $[3,4]$. The typical clinicopathologic symptoms of scrub typhus are abrupt high fever, severe headache, lymphadenopathy, generalized myalgia, eschar, and rash [5]. Accurate and effective approaches for the diagnosis of scrub typhus are lacking

\footnotetext{
* Correspondence: huanglei0248@163.com; zhangwx@hotmail.com Department of Intensive Care Unit, Peking University Shenzhen Hospital, Shenzhen 518036, People's Republic of China
}

due to the absence of typical symptoms, which often leads to misdiagnosis and underdiagnosis. A pathologic characteristic of scrub typhus is disseminated vasculitis, which can cause damage to one or more organs, such as the liver, kidney, lung, brain, meninges, and skin [6]. The clinical manifestations of scrub typhus include acute febrile illness, malaise, high fever, headache, cough, disseminated intravascular coagulation (DIC), pulmonary edema, and hepatic dysfunction [5]. However, the most typical clinical manifestation of scrub typhus is a scab at the site of the bite of the mite. Worst of all, approximately one third of the cases may suffer from multiple organ dysfunction syndrome (MODS) during the course of the disease [7]. Thrombocytopenia is another critical clinical manifestation of scrub typhus $[8,9]$, and thrombocytopenia is one of the causes of MODS [10, 11].

Scrub typhus is rare and it is hard to diagnose and treat. As far as we know, there has been only one case of scrub typhus with thrombocytopenia and MODS reported [12]. In this study, we report a second case of

(c) The Author(s). 2019 Open Access This article is distributed under the terms of the Creative Commons Attribution 4.0 International License (http://creativecommons.org/licenses/by/4.0/), which permits unrestricted use, distribution, and 
scrub typhus with thrombocytopenia and MODS and discuss its diagnosis, pathological process, and treatment. Besides, it provides a reference for the diagnosis and treatment of complex tsutsugamushi disease.

\section{Case presentation}

A 43-year-old Han Chinese woman, a sanitation worker, was admitted to our hospital after complaining of a skin infection for 7 days and sore throat with fever and dizziness for 5 days. After the onset, she took cephalosporin drugs (specific drug name and dose is unknown), the effect was not ideal. Two days before admission, she noticed a decrease in urine volume. In addition, she denied any medical history, except for gastric ulcer and angina pectoris, which were treated with unknown treatment protocols. She was married and living with her family, and she denied alcohol consumption and tobacco smoking.

At the time of admission, she was fully conscious; however, she had hypomimia. She was febrile $\left(38^{\circ} \mathrm{C}\right)$ with tachycardia (pulse 101/minute) and low blood pressure $(85 / 41 \mathrm{mmHg})$. A physical examination revealed the presence of an eschar on the right side of her neck, which was approximately $2 \mathrm{~cm} \times 1 \mathrm{~cm}$ in size (Fig. 1a).
Babinski sign, Oppenheim sign, Gordon sign, and other pathological signs were negative. A lung examination by auscultation demonstrated fine, moist rales at the base of both lungs. After careful questioning and a review of her medical history, we noticed that she had received insect bites on her neck during her sanitation work.

Laboratory examinations upon admission revealed that hemoglobin was $113 \mathrm{~g} / \mathrm{L}$, her platelet count was $21 \times 10^{9}$ platelets/L, and her white cell count was $10.09 \times 10^{9}$ cells/L $\left(8.88 \times 10^{9}\right.$ neutrophils/L, $0.74 \times 10^{9}$ lymphocytes $/ \mathrm{L}$, and $0.46 \times 10^{9}$ monocytes/L). Her coagulation function was significantly abnormal, where the prothrombin time was 24.80 (11.00-15.00 seconds), international normalized ratio was $2.25(0.80-1.20)$, D-dimer was $12.64(0-0.50 \mathrm{mg} / \mathrm{L})$, and fibrin degradation products were $52.13(0-5.00 \mathrm{mg} / \mathrm{L})$, in addition to positive plasma protamine sulfate sub-coagulation results. Her renal function was also abnormal (serum creatinine $97 \mu \mathrm{mol} / \mathrm{L}$ ). In addition, her liver enzymes were mildly elevated: serum alanine aminotransferase was 157 (9-66 $\mathrm{U} / \mathrm{L}$ ), lactate dehydrogenase was 1592 (313-618 U/L), total protein was 40.4 $(63-82 \mathrm{~g} / \mathrm{L})$, albumin was 17.2 $(35-50 \mathrm{~g} / \mathrm{L})$, serum total bilirubin was 59.7 (8.5-

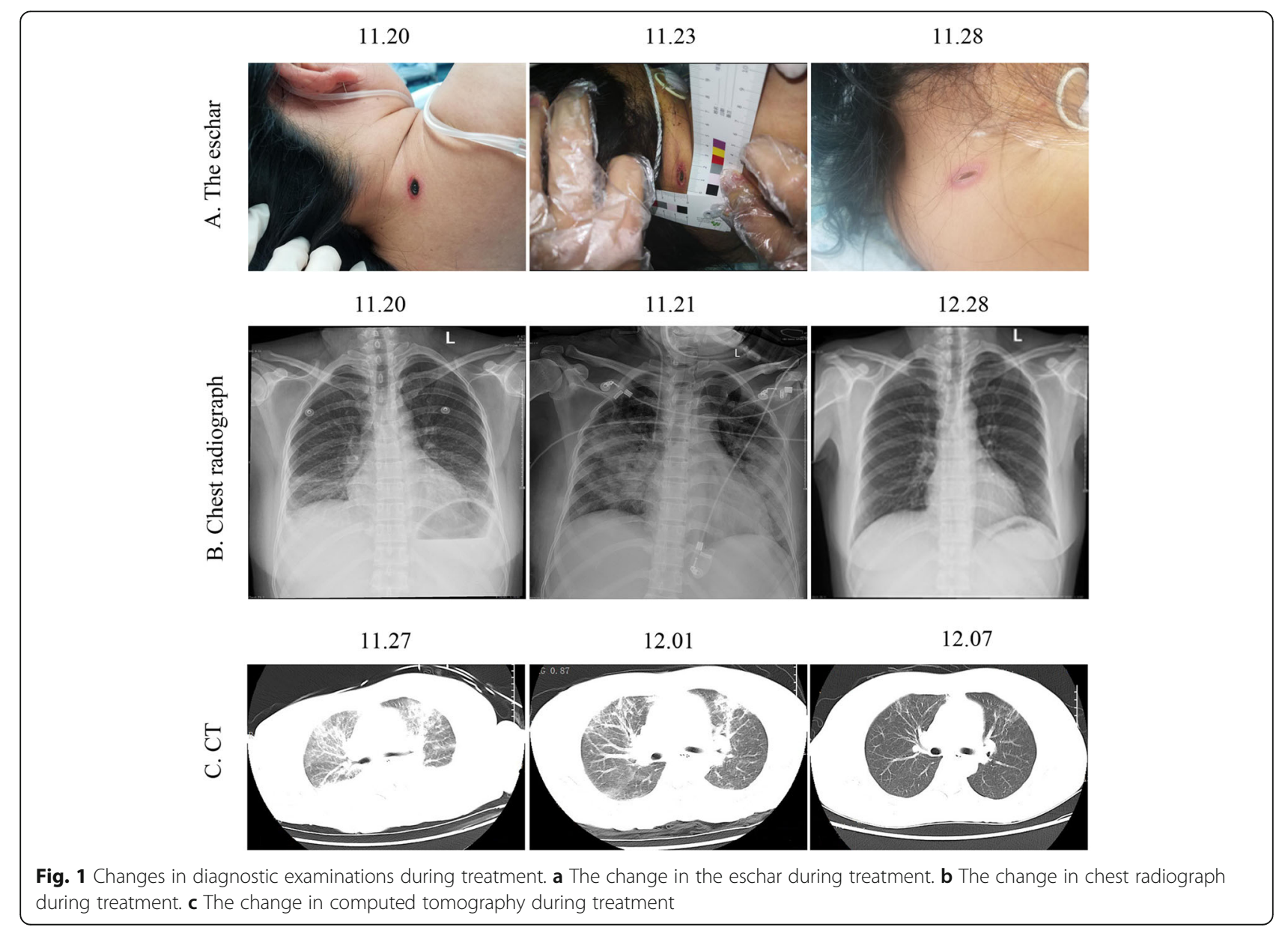


$29.2 \mu \mathrm{mol} / \mathrm{L}$ ), and serum conjugated bilirubin was 29.6 $(0-5 \mu \mathrm{mol} / \mathrm{L})$. There were also electrolyte disturbances, including hypokalemia $\left(\mathrm{Na}^{+} 127 \mathrm{mmol} / \mathrm{L}\right)$ and hyponatremia $\left(\mathrm{K}^{+} 3.40 \mathrm{mmol} / \mathrm{L}\right)$. Moreover, lactate was 3.77 (0.7-2.1 mmol/L), N-terminal pro-brain natriuretic peptide (NT-proBNP) was $555.4(<125 \mathrm{mmol} / \mathrm{L})$, and interleukin 6 was $256.20(<7.0 \mathrm{pg} / \mathrm{ml})$. Other laboratory tests did not initially show any obvious abnormalities. Chest radiographs and computed tomography $(\mathrm{CT})$ indicated bilateral pulmonary exudation (Fig. 1b, c). Her Acute Physiology, Age, Chronic Health Evaluation II (APACHE II) score was 16 , and the expected mortality rate was $8.7 \%$. Based on her clinical history as well as the laboratory and imaging examinations, the following preliminary diagnoses were made: (1) tsutsugamushi disease, (2) septic shock, and (3) thrombocytopenia of unknown origin. Imipenem/cilastatin sodium ( $1 \mathrm{~g}$ every 8 hours) was used to treat the infection, and doxycycline $(100 \mathrm{mg}$ twice a day) was used to treat scrub typhus. In addition, she received symptomatic treatment, such as platelet supplementation, liver protection, and acid inhibition.

Eight hours after admission, she developed frequent cough and complained of dyspnea. Assisted ventilation support using a non-invasive ventilator was provided. However, there was no obvious improvement in her respiratory status. On the second day of admission, she was intubated with a synchronized intermittent mandatory ventilation mode. In addition, echocardiography indicated a depressed left ventricular ejection fraction of $62 \%$ and an increased NT-proBNP that reached $1701 \mathrm{mmol} / \mathrm{L}$. Moreover, chest radiography showed that the bilateral pulmonary exudation had progressed with possible pulmonary edema. Due to her MODS (kidney, heart, lung, and brain), bedside continuous renal replacement therapy (CRRT) was given.

At that time, the Widal test, Weil-Felix test, and scrub typhus test were all negative. An human immunodeficiency virus (HIV) test, syphilis antibodies, and markers for viral hepatitis $B$ and $C$ were also negative. NT-proBNP increased to $7569 \mathrm{mmol} / \mathrm{L}$ at one time point and then returned to normal levels after symptomatic treatment. After 6 days, her vital signs were stable and the inflammatory indicators gradually decreased. Antibiotic treatment was changed to piperacillin tazobactam ( $4.5 \mathrm{~g}$ every 6 hours) and doxycycline (100 mg twice a day).

After 8 days, her platelet count increased to $200 \times 109 /$ $\mathrm{L}$, and her liver and kidney functions and infection indexes returned to normal, except for BNP $(753.9 \mathrm{mmol} /$ L). Lung auscultation detected thick breath sounds in both lungs, with no obvious dry and wet rumble. The last chest radiograph and $\mathrm{CT}$ showed that the bilateral pulmonary exudation was significantly reduced (Fig. 1b, c). Our patient became conscious and was able to breathe autonomously with grade 4 muscle strength. In addition, the eschar had nearly cleared. As a result, she was extubated. However, the Widal test and the WeilFelix test were still negative. Eleven days later, the antibiotic treatment was changed to moxifloxacin $(0.4 \mathrm{~g}$ every day) and doxycycline $100 \mathrm{mg}$ twice a day.

Fifteen days later, she recovered and was discharged. She did not experience any of the initial symptoms again, and all the indicators remained normal during the next 3 months.

\section{Discussion}

In this case, our patient's illness was caused by bug bites, which is a typical cause of tsutsugamushi disease. Because our patient did not pay enough attention to her condition, her condition worsened and finally developed into MODS with immune platelet decline. Fortunately, she received timely and accurate treatment; the prognosis of our patient is good. Compared with the existing literature reports, we draw the following summary: 1) all the causes were bug bites; and 2) the patients did not pay attention to their condition, resulting in the aggravation of their subsequent condition. However, what makes the case special is that our patient is a sanitation worker and is more likely to be bitten by bugs. Due to treatment delays, MODS occurs. However, because of the urgent condition of the patients, doctors rely on clinical experience to adopt a therapeutic diagnosis, which provides a new way for the diagnosis of complex tsutsugamushi disease.

The diagnosis of scrub typhus is a major challenge for clinicians, and as more organs are affected, the rate of misdiagnosis increases. One of the main reasons for the misdiagnosis of scrub typhus is that some clinicians are unaware of the complexity of the clinical manifestations of this disease [13, 14]. Eschar is the most important clinical manifestation for the diagnosis of scrub typhus [15], which usually forms around the swollen lymph nodes. In fact, eschars are painless, itchy, and are usually not easy to find; therefore, looking around the swollen lymph nodes usually helps to find eschars [16]. Skin ulcers on moist parts of the body, such as the perineum or armpits, are also an easily overlooked diagnostic clue for this disease [16, 17]. Nucleic acid detection, such as for HtrA (a 47-kDa periplasmic serine protease), the $56-\mathrm{kDa}$ type-specific antigen rrs (16S rRNA), and groEL (the heat shock protein Hsp60), is accurate in the early phase of infection (until 10 days after the onset of fever), after which serology becomes superior for the diagnosis of scrub typhus [18]. In addition, recent studies have revealed that surface-enhanced Raman scattering-based lateral flow assay techniques are also rapid and accurate for the diagnosis of scrub typhus [19]. Loop-mediated isothermal amplification assays are also a diagnostic method for scrub typhus, but these assays are not widely used [20]. O. tsutsugamushi immunoglobulin G (IgG) in 
blood from patients is a biomarker of scrub typhus, and the gold standard method for the diagnosis of scrub typhus is an immunofluorescence assay (IFA). Recently, with high sensitivity, specificity, and reproducibility, improved anti-Orientia immunoglobulin $M$ (IgM) and IgG-based rapid diagnostic tests and ELISAs have replaced subjective IFAs [19, 21-23]. Indirect IFAs have been the mainstay of scrub typhus diagnostics for decades, and we should pay attention to standardization, variable cutoff titers for endemic regions, and the high requirement for paired sera when using this assay [24]. In our case, no antibodies for scrub typhus were detected, and the feta test and the field test were also negative. Moreover, the laboratory indicators were atypical. The diagnosis of this disease was mainly based on the clinical manifestations (eschar, fever, and MODS) and diagnostic treatment (doxycycline treatment was effective).

There are some risk factors for determining the severity and prognosis of scrub typhus. Accumulating evidence has demonstrated that the frequency of eschars and rash in scrub typhus is closely associated with the infecting genotype [21]. In fact, $97 \%$ of all cases of eschar are caused by the Boryong genotype. On the other hand, virulent and severe diseases are usually caused by the Karp genotype [22]. Special attention should be paid to patients with scrub typhus with rapid progression that can lead to low body temperature, a rapid pulse rate, the presence of crepitation, a low percentage of lymphocytes, low serum albumin, elevated aspartate aminotransferase, elevated serum creatinine, and positive urine albumin [23].

Scrub typhus may cause thrombocytopenia and MODS. Previous studies have demonstrated that thrombotic microangiopathic syndrome is the mechanism of thrombocytopenia-associated MODS [25, 26]. A series of pathological changes such as thrombotic thrombocytopenic purpura (TTP), secondary thrombotic microangiopathy (TMA), and DIC may occur [25]. However, to the best of our knowledge, only one case of scrub typhus with thrombocytopenia and MODS has been reported thus far [12].

The early use of antibiotics is very important to avoid life-threatening scrub typhus [27], and a range of efficacious antibiotics are usually available for clinicians. In this regard, the effective antibiotics against scrub typhus are doxycycline, chloramphenicol, tetracycline, and azithromycin. Moreover, azithromycin may be more tolerable than doxycycline with the same effect [28]. On the other hand, randomized controlled trials suggested that clinicians should not use rifampicin as a first-line treatment option due to the low-certainty evidence and the risk of inducing resistance in undiagnosed tuberculosis [29].

\section{Conclusion}

In this study, we reported a case of scrub typhus with thrombocytopenia and MODS, which is only the second case worldwide. In addition, the characteristics, diagnosis, pathological process, and treatment of this disease were described and discussed in relation to previously published cases in the literature. When people suffer from scrub typhus, we should pay attention to and prevent the occurrence of MODS and immune thrombocytopenia.

\section{Acknowledgements \\ Not applicable.}

\section{Authors' contributions}

WL collection of clinical data; LH and WZ manuscript writing. All authors read and approved the final manuscript.

\section{Funding}

This study is supported by the fund of "San-ming" Project of Shenzhen (NO.SZSM 201512031).

\section{Availability of data and materials}

All data generated or analyzed during this study are included in this published article.

\section{Ethics approval and consent to participate}

The study was approved by the Ethics Committee of Peking University Shenzhen Hospital (Shenzhen, China).

\section{Consent for publication}

Written informed consent was obtained from the patient for publication of this case report and any accompanying images. A copy of the written consent is available for review by the Editor-in-Chief of this journal.

\section{Competing interests}

The authors declare that they have no competing interests.

Received: 24 March 2019 Accepted: 18 October 2019

Published online: 07 December 2019

References

1. Lee BJ, Chen CY, Hu SY, Tsan YT, Lin TC, Wang LM. Otalgia and eschar in the external auditory canal in scrub typhus complicated by acute respiratory distress syndrome and multiple organ failure. BMC Infect Dis. 2011;11:79.

2. Kelly DJ, Fuerst PA, Ching WM, Richards AL. Scrub typhus: the geographic distribution of phenotypic and genotypic variants of Orientia tsutsugamushi. Clin Infect Dis. 2009;48(Suppl 3):S203-30.

3. Taylor AJ, Paris DH, Newton PN. A systematic review of mortality from untreated scrub typhus (Orientia tsutsugamushi). PLoS Negl Trop Dis. 2015; 9(8):e0003971.

4. Bonell A, Lubell Y, Newton PN, Crump JA, Paris DH. Estimating the burden of scrub typhus: a systematic review. PLoS Negl Trop Dis. 2017;11(9):e0005838.

5. Rajapakse S, Weeratunga P, Sivayoganathan S, Fernando SD. Clinical manifestations of scrub typhus. Trans R Soc Trop Med Hyg. 2017;111(2):43-54.

6. Dogra S. Recent advances in understanding pathophysiology of scrub typhus. JK Sci. 2010;12(2):19-23.

7. Peter JV, Sudarsan TI, Prakash JA, Varghese GM. Severe scrub typhus infection: Clinical features, diagnostic challenges and management. World J Crit Care Med. 2015;4(3):244-50.

8. Lee HJ, Park CY, Park SG, Yoon NR, Kim DM, Chung CH. Activation of the coagulation cascade in patients with scrub typhus. Diagn Microbiol Infect Dis. 2017:89(1):1-6.

9. Venkategowda PM, Rao SM, Mutkule DP, Rao MV, Taggu AN. Scrub typhus: clinical spectrum and outcome. Indian J Crit Care Med. 2015;19(4):208-13.

10. Carcillo JA, Podd B, Aneja R, Weiss SL, Hall MW, Cornell TT, Shanley TP, Doughty LA, Nguyen TC. Pathophysiology of pediatric multiple organ dysfunction syndrome. Pediatr Crit Care Med. 2017;18(3_suppl Suppl 1):S32-s45. 
11. Saugel B, Phillip V, Moessmer G, Schmid RM, Huber W. Argatroban therapy for heparin-induced thrombocytopenia in ICU patients with multiple organ dysfunction syndrome: a retrospective study. Crit Care. 2010;14(3):R90.

12. Ittyachen AM, Abraham SP, Krishnamoorthy S, Vijayan A, Kokkat J. Immune thrombocytopenia with multi-organ dysfunction syndrome as a rare presentation of scrub typhus: a case report. BMC Res Notes. 2017;10(1):496

13. Jamil MD, Hussain M, Lyngdoh M, Sharma S, Barman B, Bhattacharya PK. Scrub typhus meningoencephalitis, a diagnostic challenge for clinicians: a hospital based study from North-East India. J Neurosci Rural Pract. 2015;6(4): 488-93.

14. Sharma SR, Masaraf $H$, Lynrah KG, Lyngdoh M. Tsutsugamushi disease (scrub typhus) meningoencephalitis in north eastern India: a prospective study. Ann Med Health Sci Res. 2015;5(3):163-7.

15. Arun Babu T, Vijayadevagaran V, Ananthakrishnan S. Characteristics of pediatric scrub typhus eschar in South Indian children. Pediatr Dermatol. 2017;34(2):124-7.

16. Kim DM, Won KJ, Park CY, Yu KD, Kim HS, Yang TY, Lee JH, Kim HK, Song HJ, Lee SH, et al. Distribution of eschars on the body of scrub typhus patients: a prospective study. Am J Trop Med Hyg. 2007;76(5):806-9.

17. Zhang M, Zhao ZT, Wang XJ, Li Z, Ding L, Ding SJ. Scrub typhus: surveillance, clinical profile and diagnostic issues in Shandong, China. Am J Trop Med Hyg. 2012;87(6):1099-104

18. Paris DH, Dumler JS. State of the art of diagnosis of rickettsial diseases: the use of blood specimens for diagnosis of scrub typhus, spotted fever group rickettsiosis, and murine typhus. Curr Opin Infect Dis. 2016;29(5):433-9.

19. Blacksell SD, Tanganuchitcharnchai A, Nawtaisong P, Kantipong P, Laongnualpanich A, Day NP, Paris DH. Diagnostic accuracy of the InBios scrub typhus detect enzyme-linked immunoassay for the detection of lgM antibodies in Northern Thailand. Clin Vaccine Immunol. 2016;23(2):148-54.

20. Karthikeyan PA, Hoti SL, Kanungo R. Evaluation of loop-mediated isothermal amplification assay for detection of scrub typhus in patients with acute febrile illness presenting to a Tertiary Care Center in Puducherry, India. J Lab Physicians. 2019;11(1):82-6.

21. Rodkvamtook W, Zhang Z, Chao CC, Huber E, Bodhidatta D, Gaywee J, Grieco J, Sirisopana N, Kityapan M, Lewis M, et al. Dot-ELISA rapid test using recombinant $56-k D a$ protein antigens for serodiagnosis of scrub typhus. Am J Trop Med Hyg. 2015;92(5):967-71.

22. Silpasakorn S, Srisamut N, Ekpo P, Zhang Z, Chao CC, Ching WM, Suputtamongkol Y. Development of new, broadly reactive, rapid IgG and IgM lateral flow assays for diagnosis of scrub typhus. Am J Trop Med Hyg. 2012;87(1):148-52.

23. Kingston HW, Blacksell SD, Tanganuchitcharnchai A, Laongnualpanich A, Basnyat B, Day NP, Paris DH. Comparative accuracy of the InBios scrub typhus detect lgM rapid test for the detection of IgM antibodies by using conventional serology. Clin Vaccine Immunol. 2015;22(10):1130-2.

24. Blacksell SD, Bryant NJ, Paris DH, Doust JA, Sakoda Y, Day NP. Scrub typhus serologic testing with the indirect immunofluorescence method as a diagnostic gold standard: a lack of consensus leads to a lot of confusion. Clin Infect Dis. 2007;44(3):391-401.

25. Nguyen TC, Carcillo JA. Bench-to-bedside review: thrombocytopeniaassociated multiple organ failure--a newly appreciated syndrome in the critically ill. Crit Care. 2006;10(6):235.

26. Nguyen TC, Cruz MA, Carcillo JA. Thrombocytopenia-associated multiple organ failure and acute kidney injury. Crit Care Clin. 2015;31(4):661-74

27. Wang CC, Liu SF, Liu JW, Chung YH, Su MC, Lin MC. Acute respiratory distress syndrome in scrub typhus. Am J Trop Med Hyg. 2007;76(6):1148-52.

28. Wee I, Lo A, Rodrigo C. Drug treatment of scrub typhus: a systematic review and meta-analysis of controlled clinical trials. Trans R Soc Trop Med Hyg 2017;111(8):336-44.

29. El Sayed I, Liu Q, Wee I, Hine P. Antibiotics for treating scrub typhus. Cochrane Database Syst Rev. 2018:9:Cd002150

\section{Publisher's Note}

Springer Nature remains neutral with regard to jurisdictional claims in published maps and institutional affiliations.

\section{Ready to submit your research? Choose BMC and benefit from:}

- fast, convenient online submission

- thorough peer review by experienced researchers in your field

- rapid publication on acceptance

- support for research data, including large and complex data types

- gold Open Access which fosters wider collaboration and increased citations

- maximum visibility for your research: over $100 \mathrm{M}$ website views per year

At BMC, research is always in progress.

Learn more biomedcentral.com/submissions 